\title{
Root resorption after leveling with super-elastic and conventional steel arch wires: a prospective study
}

\author{
Kawa Alzahawi ${ }^{1}$, Espen Færøvig², Pongsri Brudvik', Olav Egil Bøe ${ }^{3}$ and Maria Mavragani ${ }^{4 *}$
}

\begin{abstract}
Background: The aim of this prospective study was to compare root resorption after the leveling phase of treatment, performed by either super-elastic or conventional multi-stranded stainless steel arch wires.

Methods: From a total of 156 future orthodontic patients in a private clinic, 82 were included in the study after excluding those who earlier had orthodontic or endodontic treatment or signs of resorption. Patients were equally arbitrary allocated into two groups, where leveling was performed either with super-elastic heat-activated or conventional multi-stranded stainless steel arch wires. Root length loss was calculated using pre-treatment and post-leveling periapical radiographs.
\end{abstract}

Results: The use of super-elastic arch wires did not significantly increase the severity of root resorption, except for tooth 31, while it reduced leveling time compared to conventional stainless steel wires. Crossbite of maxillary lateral incisors seemed to be a risk factor for resorption.

Conclusion: Incisor root resorption after leveling did not differ significantly between patients treated with super-elastic and conventional stainless steel arch wires, except for a mandibular incisor.

\section{Background}

Leveling and alignment are usually the first phase of orthodontic treatment with fixed appliances. It could be considered as the 'trial' phase, when patient's cooperation and tissue responses become apparent.

Among the biological reactions to tooth movement, tendency for apical root resorption during this early phase has been shown to be indicative of the final root length loss during the entire treatment [1,2]. Even though mild apical root resorption is a usual finding among orthodontic patients [3], severe root length loss is quite infrequent. Levander and Malmgren [1] described resorption exceeding half the original root length in $1 \%$ of the tested teeth, while Linge and Linge [4] reported greater than $4 \mathrm{~mm}$ loss of root length in $2.3 \%$ of the treated teeth. Radiographs of the maxillary front teeth taken

\footnotetext{
* Correspondence: maria_mavragani@yahoo.com

${ }^{4}$ Department of Community Dentistry, Section of Orthodontics, School of Dentistry, University of Athens, Athens 10679, Greece

Full list of author information is available at the end of the article
}

after 6 months of treatment can help to identify those patients at risk of severe apical root resorption [2].

Mechanotherapy during leveling phase of treatment is performed using arch wires that fall into two broad categories: stainless steel and nickel titanium with superelastic properties. Stainless steel wires deliver a rapidly declining force, while super-elastic wires deliver a more constant force during the deactivation range [5]. Due to the convenience of the clinical use, super-elastic arch wires rapidly gained popularity among orthodontists, particularly during the leveling phase. A number of benefits for using super-elastic arch wires have been advocated; however, it has been reported that manufacturers were in such a rush to produce the ultimate aligning arch wire that very little attention has been paid to the in vivo behavior of these materials [6]. The systematic review by Riley and Bearn [7] on clinical trials on aligning arch wires advocated that there is insufficient data to make clear recommendations regarding the most effective arch wire for alignment. In a later Cohrane review by Wang et al. [8], some additional factors, including the amount of root resorption along with tooth movement and the intensity of 
pain experienced by patients during initial alignment, were evaluated. The authors concluded that there is some evidence to suggest that there is no difference between the speed of tooth alignment or pain experienced by patients when using one initial aligning arch wire over another. However, as they report, root resorption had not been investigated by any randomized clinical trial, even though it is one of the most serious side effects of orthodontic treatment. They suggest that further evaluation of the aligning arch wires should consider this potentially serious side effect of orthodontic treatment.

As derived from the literature, there seems to be lack of knowledge concerning the effect of the leveling arch wires used in everyday clinical practice on orthodontic root resorption. Therefore, the primary objective of this study was to compare the amount of root resorption following the leveling phase of orthodontic treatment performed with super-elastic arch wires and conventional multi-stranded stainless steel arch wires. Leveling duration was compared between the groups. Additionally, a comparison of the severity of apical root resorption between central and lateral incisors during that phase of treatment was attempted. Finally, the effect of several patient- and treatment-related variables on apical root resorption was evaluated.

\section{Methods}

The study was approved by both the Ethical Committee in Norway and the Norwegian Social Science Data Services (NSD) (REK Vest 229.08 2008/12408-ØYSV). All study participants and/or their parents read and signed an informed consent form before treatment initiation. A power analysis was performed for calculation of the sample size. In order to identify a difference of $0.5 \mathrm{~mm}$ at the 0.05 level of significance with $80 \%$ power, using a standard deviation (SD) of 0.8, 41 patients were required in each group.

One hundred fifty-six orthodontic patients, who were about to start treatment at the private clinic of one of the authors (EF), were assessed for eligibility to participate in the study. Patients who showed signs of apical root resorption, were earlier orthodontically treated, or had endodontic treatment of their front teeth were excluded from the sample. Finally, 82 patients were included in this prospective study.

All patients received orthodontic treatment with straight-wire edgewise technique. The appliances used were 0.022-in. Diamond Twin brackets (Ormco Corp.,
Clendora, CA, USA). The study was parallel in design, with an allocation ratio of 1:1. Clinical assistants arbitrarily allocated patients into two experimental groups with 41 patients each, using prepared random number list (Table 1). Treatment was performed by one of the authors (EF), who was informed about the treatment group during bonding. During the leveling phase, patients in the first group were treated with super-elastic arch wires (SE group), whereas patients in the second group were treated with stainless steel arch wires (SS group). The typical arch wire sequence for the SE group was 0.014 in. Sentalloy, 0.016 in. Sentalloy, and $0.018 \times 0.025$ in. Bioforce (Dentsply/GAC, Bohemia, NY, USA). The arch wire sequence for the SS group was 0.0175 in. Penta-one multi-stranded (Masel Orthodontics, Carlsbad, CA, USA), 0.016 in. Australian regular (A J Wilcock PTY, LTD, Whittlesea, Australia), and $0.016 \times$ 0.022 in. resilient (3M Unitek, Monrovia, CA, USA).

A number of variables were recorded for each patient (gender, age, ANB angle, overjet, overbite, impacted maxillary canines, invagination of each incisor, crossbite separately for teeth 12 and 22). During the course of treatment, any complaint from the patients would be noted in their clinical journal. All patients were radiographically examined at two predetermined points of treatment: before treatment start and at the end of the leveling phase, approximately 6 to 10 months after initiation of treatment. Leveling duration was also recorded. During the examinations, periapical radiographs of maxillary and mandibular incisor teeth were taken with a standardized long-cone paralleling technique. The tube was placed at a fixed distance of $7 \mathrm{~cm}$ from the film, using a customized Eggen holder. Four radiographs were taken each time: one with the central ray beam between the two maxillary central incisors, one with the X-ray beam centered at the maxillary lateral incisor on each side, and one radiograph with the central beam between the mandibular central incisors. Both apex and the incisal edge of the incisor teeth should be clearly visible. Unsatisfactory X-rays were excluded from the study. Totally, 311 teeth were examined radiographically in 79 patients: 2 teeth in 6 patients, 3 in 18 patients, 4 in 46 patients, 5 in 1 patient, 6 teeth in 1 patient, 7 teeth in 6 patients, and finally 8 teeth in 1 patient. All periapical radiographs were converted to digital images by using an EPSON perfectionV700 scanner at a resolution of 300 dpi (Epson Inc., Long Beach, CA, USA). The

Table 1 Gender and age distribution of the patients in the two groups

\begin{tabular}{|c|c|c|c|c|c|c|}
\hline & Males & Females & All & Mean age (years) & Range (years) & $\overline{S D}$ \\
\hline SE group & 22 & 19 & 41 & 13.3 & 11 to 16 & 1.5 \\
\hline SS group & 18 & 23 & 41 & 13.7 & 11 to 22 & 2.4 \\
\hline All & 40 & 42 & 82 & 13.5 & 11 to 22 & 2.0 \\
\hline
\end{tabular}


Windows-based CliniView software package (Instrumentarium Dental, Tuusula, Finland) was used for adjustment in brightness and contrast between scanned images, and for measurements.

\section{Analysis of radiographs}

Root resorption has been estimated as any reduction in the radiographic length of all incisors from the cementoenamel junction (CEJ) to the root apex, after adjustment for different magnifications. The anatomic landmarks were demarcated on the X-ray films. For CEJ, the most distinct landmark either mesial or distal was used, but once selected, the same side was used for all radiograms of the tooth. The longitudinal axis of each tooth was constructed following the root canal. The marked CEJ, root apex, and incisal edge were projected perpendicular to this axis. Root length was measured from the projected CEJ to the apex on the constructed longitudinal axis, and crown length similarly to the incisal edge (Figure 1). A correction factor was calculated in order to relate the pre-treatment and post-leveling radiographs to each other. Root length was adjusted by using the crown length registrations, assuming crown length to be unchanged over the observation period. This method has earlier been described [9]. Its accuracy has been found satisfactory [10].

\section{Measurement error}

All measurements were performed by one examiner (KA), who was blinded regarding the origin of the radiographs. The reproducibility of the measurements was assessed by analyzing the difference between double measurements by the same examiner on radiographs taken at least 2 weeks apart on 4 teeth (totally 72 teeth), one tooth on each side, both in the maxilla and mandible in 18 randomly selected patients. The systematic error between the double

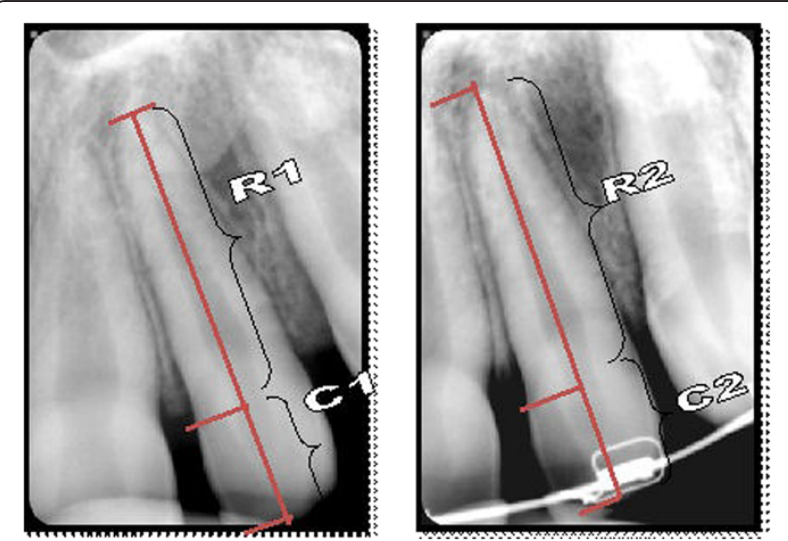

Figure 1 Measurements on pre-treatment and post-leveling radiograms. $\mathrm{C} 1$, crown length on pre-treatment radiogram, C2, crown length on post-leveling radiogram; R1, root length before treatment; R2, root length after leveling phase. measurements was evaluated separately for each root length using the paired $t$ test, and the measurement error on a single measurement was estimated by using the formula $\tau=\mathrm{SD} / \sqrt{2}$, where SD is the standard deviation of the differences between duplicated measurements.

\section{Statistical methods}

In order to investigate whether the recorded variables, including leveling duration, differed significantly between the two treatment groups, a two-sample $t$ test was carried out for the quantitative variables and a chisquare test for the qualitative variables. A two-sample $t$ test was also used to test for any difference in root length reduction in millimeters and percentage between the two groups, for each examined tooth $(12,11,21,22$, 32, 31, 41, and 42) separately.

To test for differences in root resorption between the central and lateral incisors, a paired $t$ test was performed on the values of the central and the lateral incisors of the same quadrant of each patient. Finally, in order to assess any association between the percentage root shortening of each tooth and the other variables recorded, multiple linear regression analysis was applied. The statistical analyses were carried out using the SPSS software package (SPSS Inc., IBM Company, Chicago, IL, USA).

\section{Results}

None of the 41 allocated patients in each group was either lost to follow-up or discontinued. Finally, 41 patients in each group were analyzed (Figure 2). Patients' recruitment was performed during fall 2009, and the follow-up lasted until spring/summer 2010.

The two patient groups were well matched for all treatment variables except for leveling duration which was longer $(P=0.002 ; 95 \% \mathrm{CI}=-1.31$ to -0.30$)$ for patients

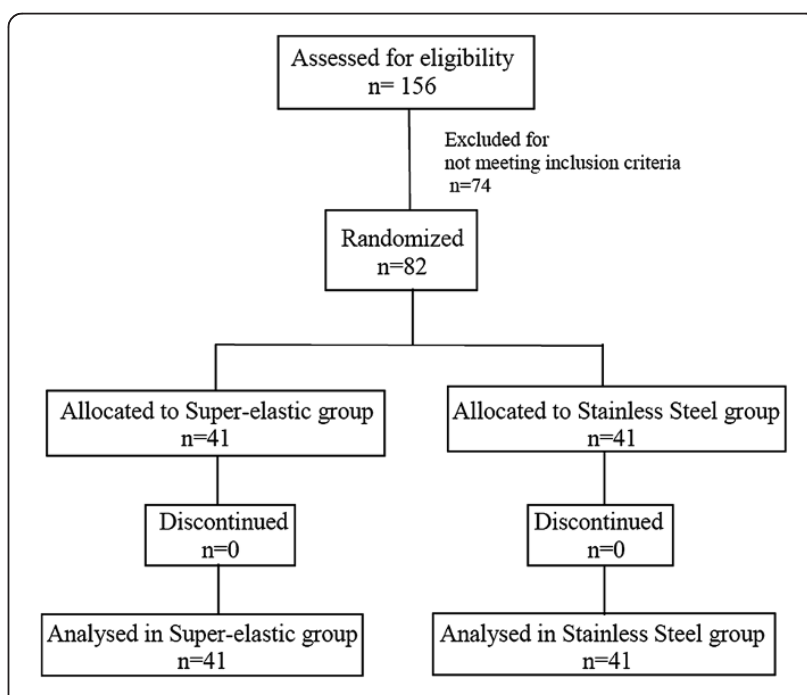

Figure 2 CONSORT flowchart diagram of the clinical trial. 
treated with stainless steel arch wires (mean 6.98 months, SD 1.42) than for patients treated with super-elastic arch wires (mean 6.17 months, SD 0.77), and invagination. Chisquare test revealed more invaginated incisors at the left side for the group treated with stainless steel than the one treated with super-elastic arch wires (tooth 21 (invaginated teeth out of a total of 41): super-elastic group 2, stainless steel group $11, P=0.005$; tooth 22 (invaginated teeth out of a total of 41): super-elastic group 4, stainless steel group $11, P=0.04)$. No significant differences were revealed between groups for agenesis, trauma, and crossbites. Similarly, ANB angle (super-elastic: mean 2.8 SD 2.15; stainless steel: mean $3.45^{\circ}$, SD 1.98; $P=0.85$ ), overjet (super-elastic: mean $4.73 \mathrm{~mm}$, SD 2.90; stainless steel: mean $4.68 \mathrm{~mm}$, SD 2.77; $P=0.91$ ), and overbite (super-elastic: mean $3.63 \mathrm{~mm}$, SD 0.34; stainless steel: mean 3.61, SD 0.38; $P=0.52$ ) did not differ between the two groups.

Statistical analysis of the difference in root resorption between the two treatment groups, in millimeters and in percentage of root shortening, for each tooth, revealed no significant results, except for tooth 31 that showed more resorption in the group treated with super-elastic arch wires (Table 2). Negative values, indicating root lengthening, were noted in most of the tooth groups. There was a tendency for more root resorption for the lateral than the central incisors both in the maxilla and mandible, with significant difference only between teeth 41 and 42 (Table 3).

The results of the regression analysis, with the percentage of root shortening of the upper lateral incisors as dependent variable and all other variables as independent variables, revealed that apical root resorption of maxillary lateral incisors was significantly associated with the presence of crossbite of these teeth (Table 4). No other significant association between apical root resorption after leveling and other variables examined was shown for any tooth.

No complaints for harm or unintended effects from patients under treatment were reported.

Table 2 Root resorption in millimeters and in percentage of the initial root length in the two groups

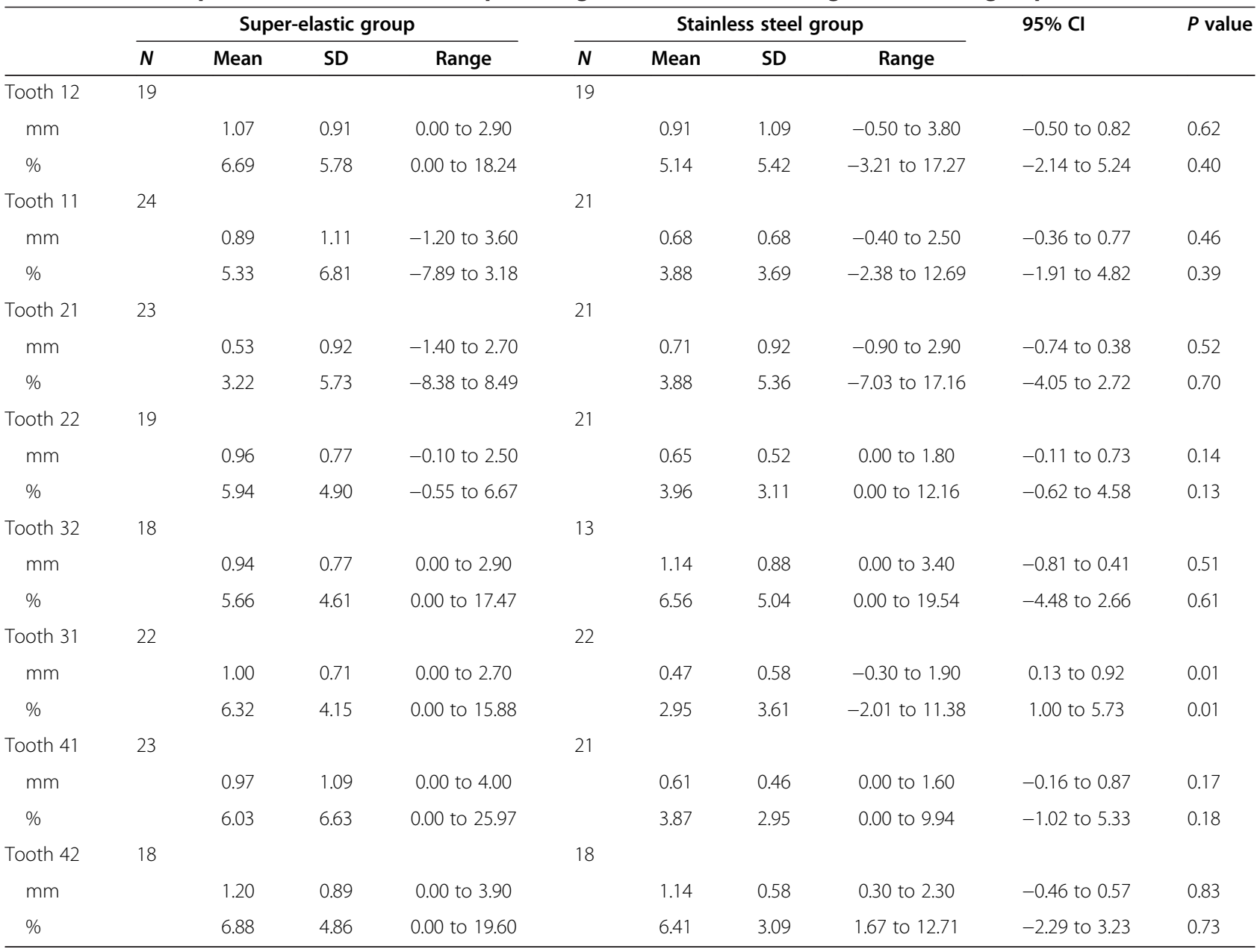


Table 3 Root resorption in millimeters and percentage of initial root length for lateral and central incisors in maxilla and mandible

\begin{tabular}{|c|c|c|c|c|c|c|c|c|c|c|c|c|}
\hline & Mean & SD & Mean & SD & $95 \% \mathrm{Cl}$ & $P$ & Mean & SD & Mean & SD & $95 \% \mathrm{Cl}$ & $\bar{P}$ \\
\hline \multirow[t]{2}{*}{ Pairs } & \multicolumn{2}{|c|}{12} & \multicolumn{2}{|c|}{11} & & & \multicolumn{2}{|c|}{22} & \multicolumn{2}{|c|}{21} & & \\
\hline & \multicolumn{4}{|c|}{$(N=37)$} & & & \multicolumn{4}{|c|}{$(N=37)$} & & \\
\hline $\mathrm{mm}$ & 0.97 & 1.00 & 0.74 & 0.87 & -0.13 to 0.60 & 0.20 & 0.71 & 0.57 & 0.62 & 0.94 & -0.23 to 0.42 & 0.55 \\
\hline$\%$ & 5.79 & 5.60 & 4.30 & 5.02 & -0.54 to 3.52 & 0.15 & 4.36 & 3.45 & 3.42 & 5.57 & -1.04 to 2.92 & 0.34 \\
\hline \multirow[t]{2}{*}{ Pairs } & \multicolumn{2}{|c|}{42} & \multicolumn{2}{|c|}{41} & & & \multicolumn{2}{|c|}{32} & \multicolumn{2}{|c|}{31} & & \\
\hline & \multicolumn{4}{|c|}{$(N=36)$} & & & \multicolumn{4}{|c|}{$(N=30)$} & & \\
\hline $\mathrm{mm}$ & 1.17 & 0.74 & 0.73 & 0.72 & 0.21 to 0.68 & $<0.01$ & 1.04 & 0.82 & 0.79 & 0.65 & -0.08 to 0.58 & 0.14 \\
\hline$\%$ & 6.65 & 4.02 & 4.54 & 4.22 & 0.75 to 3.47 & $<0.01$ & 6.14 & 4.78 & 5.07 & 4.02 & -0.88 to 3.01 & 0.27 \\
\hline
\end{tabular}

\section{Discussion}

Only a limited number of teeth from each incisor planned to be examined were finally analyzed due to inappropriate radiographs. That may have a negative effect on the power of the study, since statistical analysis was performed for each tooth separately. This drawback could have been eliminated by providing additional training to the dental assistants for the specific requirements of the particular $\mathrm{X}$-rays, mainly related to the full appearance of the incisal edge of the teeth.

The two groups did not differ considering the characteristics of malocclusion recorded, except for invagination for the left incisor teeth. Mild type of invagination, however, has been shown not to be a risk factor for orthodontic root resorption [11]. The severity of crowding has not been considered at this study since crowding determines to a large extent the amount of tooth movement during leveling, which could have influenced the results.

Negative values for root resorption indicating root length increase have also previously been reported and attributed either to a real increase in root length $[4,12]$ or to method error [13]. Error in registration of the CEJ, which is difficult to be identified, should also be considered.

The present study was performed in a sample of Norwegian orthodontic patients who were treated with straight-wire edgewise technique and conventional brackets. It could be assumed that the results from this study could be applicable for any patient treated with the same technique.

The end of leveling phase has been chosen as the time point for the evaluation of the effect of the two different arch wire materials, due to the possibility to use one type of arch wire material only, before the continuation of treatment that would require both types of arch wires. Additionally, the amount of root shortening 6 to 12 months after bracket placement is of high predictive value for the severity of root resorption after the completion of treatment [2]. Incisors were selected for this study because they are the most prone to tooth resorption, while a small reduction of their length is easier to be detected by conventional radiography. Most of the patients included in this study (90\% of the examined teeth) exhibited signs of root resorption after initial treatment of 6 to 10 months. For the majority of the cases, the amount of resorption was minor, but present. According to Makedonas et al. [14], root resorption diagnosed by cone beam computed tomography after 6 months of treatment was clinically significant only for the $4 \%$ of the patients.

Leveling was accomplished sooner by super-elastic than stainless steel arch wires in our sample. Similarly, Weiland [15] has shown greater amount of buccal premolar

Table 4 Regression analysis for teeth 12 and 22

\begin{tabular}{|c|c|c|c|c|c|c|c|c|}
\hline & \multicolumn{4}{|c|}{ Tooth 12} & \multicolumn{4}{|c|}{ Tooth 22} \\
\hline & Constant & Crossbite 12 & Age & $R^{2}$ (adjusted) \% & Constant & Crossbite 22 & ANB & $R^{2}$ (adjusted) \% \\
\hline \multirow[t]{2}{*}{1} & 0.783 & 0.967 & & 13.9 & 0.675 & 0.600 & & 11.3 \\
\hline & & 0.012 & & & & 0.019 & & \\
\hline \multirow[t]{2}{*}{2} & -1.149 & 1.052 & 0.143 & 17.6 & 0.975 & 0.492 & -0.091 & 16.8 \\
\hline & & 0.006 & 0.116 & & & 0.052 & 0.060 & \\
\hline $95 \% \mathrm{Cl}$ & & 0.32 to 1.79 & -0.04 to 0.32 & & & 0.00 to 0.99 & -0.19 to 0.01 & \\
\hline
\end{tabular}

The figures in the first column denote the number of explanatory variables included in the regression analysis by first applying the best subset regression analysis among all variables. For each regression analysis, the partial regression coefficient of the variable is given in bold and the $P$ value of the partial regression coefficient in italics. 
displacement during the same period with super-elastic than stainless steel wires. Initial force applied by stainless steel wires soon declines and needs to be re-established, while the force induced by means of super-elastic wires continues over a longer time. Tooth movement with continuous forces was also found more effective than with interrupted continuous forces at the premolar tipping model by Owman-Moll et al. [16]. Longer treatment time with steel wires could be attributed to the intervals when steel wires are passive. However, in the Cochrane review of published randomized controlled trials (RCTs) by Wang et al. [8], no difference in the speed of tooth alignment could be shown after using one initial aligning arch wire over another. Authors commented on the general poor quality of the included trials and suggested that the results should be viewed with caution.

At the same Cohrane review, the lack of RCTs on the effect of different types of aligning arch wires on the severity of the induced root resorption was emphasized. At the experimental premolar buccal tipping by Weiland [15], root resorptive activity was found lower for the teeth moved by steel than super-elastic wires. The present study evaluated root resorption during the first phase of orthodontic treatment. Any type of tooth movement necessary for alignment was performed. No statistically significant difference was found between super-elastic and multi-stranded steel arch wires, except for the lower left central incisor which seemed to be significantly more affected by root resorption in the group treated with superelastic arch wires. A general tendency for more severe apical root resorption in the super-elastic group was noticed, but that was only significant for tooth 31 . Treatment duration that has been considered a risk factor for orthodontically induced root resorption [17-19] was longer for the stainless steel group. It could be hypothesized that the inactive intervals at force application, which may prolong treatment time, allow for repair of resorption lacunae. When orthodontic force is discontinued or falls under a certain level, lacunae are repaired by a process that is similar to the early cementogenesis occurring during tooth development $[20,21]$. In other experimental animal studies, allowing for inactive periods of force application was beneficial against root resorption [22-24].

No significant difference in root resorption between the central and lateral incisors was revealed, except for the lower right incisors. There was a tendency, however, for more root resorption for the lateral incisors compared with the central incisors. Recent studies by Nanekrungsan et al. [25] and Lund et al. [3] found lateral incisors to be more often and seriously affected by root resorption.

An interesting finding from this study was the significant relation between crossbite and root resorption of the maxillary lateral incisors. That was not in agreement with Kaley and Phillips [26] who did not find any significant relation between crossbite and apical root resorption. In that study, the evaluation was performed at the end of orthodontic treatment, after the additive to the presently studied initial resorption effect of the subsequent phases of treatment. However, the authors recognized root torquing as risk factor for orthodontic resorption. The relation between crossbite and root resorption found in this study could be attributed to the increased amount of load at the apical thin area, when the tipping movement for the correction of the crossbite occurs. Teeth may be more prone to apical resorption during tipping than bodily movement, where pressure is more or less evenly distributed on the buccal and the lingual surfaces of the root [27]. It could also be possible that lateral incisors may had been subjected to traumatizing occlusal forces during the correction of the anterior crossbite, since the bite has not been raised to facilitate the correction of the crossbite in any of the cases. Even though Newman [28] did not find any relationship between root resorption and occlusal trauma or heavy occlusal forces, one could keep in mind that these factors can create jiggling movements. The latter has been proven to be a considerable risk factor for orthodontic root resorption [29].

\section{Conclusion}

Root resorption of maxillary and mandibular incisors after leveling did not statistically differ among patients treated with super-elastic and those being treated with conventional stainless steel arch wires, except for the mandibular left central incisor that showed more extensive resorption in the super-elastic group. Crossbite seemed to be a risk factor for root resorption of the maxillary lateral incisors, during the initial stages of orthodontic treatment.

Competing interests

The authors declare that they have no competing interests.

\section{Authors' contributions}

KA conceived the study, performed measurements, scientific writing. EF conceived the study, carried-out patients' treatment in his private practice. PB participated in study design, administration of ethical permision process and contributed to the scientific content of the study. OEB participated in study design, carried-out the statistical analysis. MM coordinated the study, participated in study design, statistical evaluation and scientific writing.

\section{Author details}

'Department of Clinical Dentistry-Orthodontics, Faculty of Medicine and Dentistry, University of Bergen, Årstadveien 19/21, Bergen 5009, Norway. ${ }^{2}$ Department of Orthodontics, Dental Faculty, University of Oslo, Oslo 0316, Norway. ${ }^{3}$ Department of Clinical Dentistry, Faculty of Medicine and Dentistry, University of Bergen, Bergen 5009, Norway. ${ }^{4}$ Department of Community Dentistry, Section of Orthodontics, School of Dentistry, University of Athens, Athens 10679, Greece

Received: 23 January 2014 Accepted: 8 April 2014

Published online: 15 May 2014 


\section{References}

1. Levander $E$, Malmgren $O$. Evaluation of the risk of root resorption during orthodontic treatment: a study of upper incisors. Eur J Orthod. 1988; 10:30-8.

2. Artun J, Ron Van't H, Doppel D, Jagtman K. Identification of orthodontic patients at risk of severe apical root resorption. Am J Orthod Dentofacial Orthop. 2009; 135:448-55.

3. Lund H, Gröndahl K, Hansen K, Gröndahl HG. Apical root resorption during orthodontic treatment: a prospective study using cone beam $\mathrm{CT}$. Angle Orthod. 2012; 82:480-7.

4. Linge $L$, Linge $B O$. Patient characteristics and treatment variables associated with apical root resorption during orthodontic treatment. Am J Orthod Dentofacial Orthop. 1991; 99:35-43.

5. Miura F, Mogi M, Ohura Y, Hamanaka H. The super-elastic property of the Japanese NiTi alloy wire for use in orthodontics. Am J Orthod Dentofacial Orthop. 1986; 90:1-10.

6. Evans TJ, Durning P. Aligning archwires, the shape of things to come? A fourth and fifth phase of force delivery. Br J Orthod. 1996; 23:269-75.

7. Riley M, Bearn DR. A systematic review of clinical trials of aligning archwires. J Orthod. 2009; 36:42-51.

8. Wang Y, Jian F, Lai W, Zhao Z, Yang Z, Liao Z, Shi Z, Wu T, Millett DT, McIntyre GT, Hickman J. Initial arch wires for alignment of crooked teeth with fixed orthodontic braces (Review). Cochrane Database Syst Rev. 2010; 4(4):CD007859.

9. Mavragani M, Vergari A, Selliseth NJ, Bøe OE, Wisth PJ. A radiographic comparison of apical root resorption after orthodontic treatment with a standard edgewise and a straight-wire edgewise technique. Eur J Orthod. 2000; 22:665-74

10. Blake M, Woodside DG, Pharoah MJ. A radiographic comparison of apical root resorption after orthodontic treatment with the edgewise and Speed appliances. Am J Orthod Dentofacial Orthop. 1995; 108:76-84.

11. Mavragani M, Apisariyakul J, Brudvik P, Selvig KA. Is mild dental invagination a risk factor for apical root resorption in orthodontic patients? Eur J Orthod. 2006; 28:307-12.

12. Mavragani $M, B ø e ~ O E$, Wisth PJ, Selvig KA. Changes in root length during orthodontic treatment: advantages for immature teeth. Eur J Orthod. 2002; 24:91-7

13. Mirabella $A D$, Årtun J. Risk factors for apical root resorption of maxillary anterior teeth in adult orthodontic patients. Am J Orthod Dentofacia Orthop. 1995; 108:48-55.

14. Makedonas D, Lund H, Grondahl K, Hansen K. Root resorption diagnosed with cone beam tomography after 6 months of orthodontic treatment with fixed appliance and the relation to risk factors. Angle Orthod. 2012; 82:196-201

15. Weiland F. Constant versus dissipating forces in orthodontics: the effect on initial tooth movement and root resorption. Eur J Orthod. 2003; 25:335-42.

16. Owman-Moll P, Kurol J, Lundgren D. Continuous versus interrupted continuous orthodontic force related to early tooth movement and root resorption. Angle Orthod. 1995; 65:395-401.

17. Baumrind $\mathrm{S}$, Korn EL, Boyd RL. Apical root resorption in orthodontically treated adults. Am J Orthod Dentofacial Orthop. 1996; 110:311-20.

18. Taithingchai R, Sookkorn K, Killiany DM. Facial and dentoalveolar structure and the prediction of apical root shortening. Am J Orthod Dentofacial Orthop. 1996; 110:296-302.

19. Jiang RP, McDonald JP, Fu MK. Root resorption before and after orthodontic treatment: a clinical study of contributory factors. Eur $J$ Orthod. 2010; 32:693-7.

20. Rygh P. 1977 Orthodontic root resorption studied by electron microscopy. Angle Orthod. 1977; 47:1-16.

21. Brudvik P, Rygh P. The repair of orthodontic root resorption: an ultrastructural study. Eur J Orthod. 1995; 17:189-98.

22. Maltha JC, van Leeuwen EJ, Dijkman GEHM, Kuijpers-Jagtman AM. Incidence and severity of root resorption in orthodontically moved premolars in dogs. Orthod Craniofacial Res. 2004; 7:115-21.
23. Kameyama T, Matsumoto $\mathrm{Y}$, Warita H, Soma K. Inactivated periods of constant orthodontic forces related to desirable tooth movement in rats. J Orthod. 2003; 30:31-7.

24. Gu G, Lemery SA, King GJ. Effect of appliance reactivation after decay of initial activation on osteoclasts, tooth movement, and root resorption. Angle Orthod. 1999; 69:515-22.

25. Nanekrungsan K, Patanaporn V, Janhom A, Korwanich N. External apical root resorption in maxillary incisors in orthodontic patients: associated factors and radiographic evaluation. Imaging Sci Dent 2012; 42:147-54.

26. Kaley J, Phillips C. Factors related to root resorption in edgewise practice. Angle Orthod. 1991; 61:125-32.

27. Reitan K. Initial tissue behavior during apical root resorption. Angle Orthod. 1974; 44:68-82

28. Newman WG. Possible etiologic factors in external root resorption. Am Orthod Dentofacial Orthop. 1975; 67:522-39.

29. Linge $B O$, Linge L. Apical root resorption in upper anterior teeth. Eur J Orthod. 1983; 5:173-83.

doi:10.1186/s40510-014-0035-z

Cite this article as: Alzahawi et al:: Root resorption after leveling with super-elastic and conventional steel arch wires: a prospective study. Progress in Orthodontics 2014 15:35.

\section{Submit your manuscript to a SpringerOpen ${ }^{\odot}$ journal and benefit from:}

- Convenient online submission

- Rigorous peer review

- Immediate publication on acceptance

- Open access: articles freely available online

- High visibility within the field

- Retaining the copyright to your article

Submit your next manuscript at $>$ springeropen.com 